Zygote 24 (February), pp. 89-97. (c) Cambridge University Press 2015. This is an Open Access article, distributed under the terms of the Creative Commons Attribution licence (http://creativecommons.org/licenses/by/3.0/), which permits unrestricted re-use, distribution, and reproduction in any medium, provided the original work is properly cited.

doi:10.1017/S0967199414000768 First Published Online 12 February 2015

\title{
ING2 (inhibitor of growth protein-2) plays a crucial role in preimplantation development
}

\author{
Lin Zhou ${ }^{2}$, Pei Wang ${ }^{2}$, Juanjuan Zhang ${ }^{2}$, Boon Chin Heng ${ }^{3,4}$ and Guo Qing Tong ${ }^{1,5}$ \\ Nanjing Maternity and Child Health Care Hospital Affiliated to Nanjing Medical University, Nanjing, PR China; Sunway \\ University, Bandar Sunway, Selangor Darul Ehsan, Malaysia; D-BSSE, ETH-Zurich, Basel, Switzerland; and Shuguang \\ Hospital Affiliated to Shanghai University of Traditional Chinese Medicine, Shanghai, PR China
}

Date submitted: 13.07.2014. Date revised: 10.10.2014. Date accepted: 17.11.2014

\section{Summary}

\begin{abstract}
ING2 (inhibitor of growth protein-2) is a member of the ING-gene family and participates in diverse cellular processes involving tumor suppression, DNA repair, cell cycle regulation, and cellular senescence. As a subunit of the Sin3 histone deacetylase complex co-repressor complex, ING2 binds to H3K4me3 to regulate chromatin modification and gene expression. Additionally, ING2 recruits histone methyltransferase (HMT) activity for gene repression, which is independent of the HDAC class I or II pathway. However, the physiological function of ING2 in mouse preimplantation embryo development has not yet been characterized previously. The expression, localization and function of ING2 during preimplantation development were investigated in this study. We showed increasing expression of ING2 within the nucleus from the 4-cell embryo stage onwards; and that down-regulation of ING2 expression by endoribonuclease-prepared small interfering RNA (esiRNA) microinjection results in developmental arrest during the morula to blastocyst transition. Embryonic cells microinjected with ING2-specific esiRNA exhibited decreased blastulation rate compared to the negative control. Further investigation of the underlying mechanism indicated that down-regulation of ING2 significantly increased expression of $p 21$, whilst decreasing expression of HDAC1. These results suggest that ING2 may play a crucial role in the process of preimplantation embryo development through chromatin regulation.
\end{abstract}

Keywords: ING2, Inhibitor of growth protein, Preimplantation development

\footnotetext{
${ }^{1}$ All correspondence to: Guo Qing Tong. Reproduction Medicine Center, Shuguang Hospital Affiliated to Shanghai University of Traditional Chinese Medicine, 528 Zhangheng Rd, Shanghai 201203, PR China. e-mail: tongguoqing@hotmail.com

${ }^{2}$ State Key Laboratory of Reproductive Medicine, Department of Reproduction, Nanjing Maternity and Child Health Care Hospital Affiliated to Nanjing Medical University, Nanjing 210004, PR China.

${ }^{3}$ Sunway University, Department of Biological Sciences, Faculty of Science \& Technology, 5 Jalan Universiti, Bandar Sunway 47500, Selangor Darul Ehsan, Malaysia.

${ }^{4}$ Department of Biosystems Science \& Engineering (D-BSSE), ETH-Zurich, Mattenstrasse 26, Basel 4058, Switzerland.

${ }^{5}$ Reproduction Medicine Center, Shuguang Hospital Affiliated to Shanghai University of Traditional Chinese Medicine, 528, Zhangheng Rd, Shanghai 201203, PR China.
}

\section{Introduction}

Preimplantation embryo development includes fertilization, cell cleavage, morula and blastocyst formation. The formation of a healthy blastocyst is critical for subsequent implantation, pregnancy and fetal development (Watson \& Barcroft, 2001). The preimplantation embryo exhibits an autonomous development capability influenced by various factors within the maternal and embryonic environment (Schultz, 2005). Similarly, in vitro culture conditions also exert crucial effects on embryo developmental potential (Niemann \& Wrenzycki, 2000; Schultz \& Williams, 2002; Ecker et al., 2004). In clinical assisted reproductive technology, embryos are selected for transfer based mainly on morphological criteria, which to some extent reflect their developmental potential. In most cases, day- 2 or day- 3 cleavage-stage embryos are usually transferred. Human embryonic genome 
activation (EGA) occurs from the 4-8-cell stage (Braude et al., 1988), which makes embryo selection at earlier developmental stages less reliable. In reality, prolonged culture is not always utilized as a selection tool because aneuploidies or aberrant gene expression prevent development to the blastocyst stage in a large proportion of embryos (Gardner et al., 1998; Dal Canto et al., 2012). Hence, it is imperative to characterize the mechanisms controlling preimplantation development, to further our understanding of basic reproductive biology, as well as advance practical applications in the clinic.

Preimplantation embryonic development is regulated both genetically and epigenetically. The global gene expression profile is dynamic and displays stage-specific characteristics at different developmental stages of the preimplantation embryo. For example, Oct4, Nanog, Gata3 and Bmp8b are activated between the 4-8-cell embryonic stage (Hamatani et al., 2004). These genes are key regulators of differentiation into the inner cell mass (ICM) and trophectoderm (TE), which is the very first cell lineage specification in mouse embryonic development (Yamanaka et al., 2006). Many studies have suggested that DNA methylation and the regulation of histone modifications have profound effects on preimplantation embryonic development (Monk et al., 1987; Santos et al., 2005; Yeo et al., 2005; TorresPadilla et al., 2006). Two major histone deacetylase (HDAC) complexes (NuRD and SIN3) have specific functions in embryonic development (Ahringer, 2000). HDAC1 is a major deacetylase in preimplantation embryos and its expression negatively regulates the acetylation state of histone H4K5 during preimplantation embryonic development (Ma \& Schultz, 2008). Although knowledge of the mechanisms of preimplantation development has increased gradually over the past decade, the key transcription factors that are essential for blastocyst formation and how they interact with epigenetic regulators have yet to be characterized.

ING2 participates in diverse cellular processes involving tumor suppression, DNA repair, cell cycle regulation, and cellular senescence, all of which are functionally linked to the p53 tumor suppressor protein (Unoki et al., 2008; Zhang et al., 2008; Larrieu et al., 2009). Additionally, ING2 has biological functions that are independent of the p53 pathway. As a basic subunit of the histone deacetylase Sin 3 complex, ING2 mediates the binding of the Sin 3 complex to some promoters where di-/tri-methylated H3K4 regulates gene repression (Smith et al., 2010). ING2 can also play a role in modulating histone methyltransferase (HMT) activity associated with silencing function, which involves a non-HDAC class I or II pathway (Goeman et al., 2008). As ING2 modulates both gene expression and chromatin modification, this study attempted to examine the role of ING2 in mouse preimplantation embryonic development.

\section{Materials and methods}

All chemicals and culture media were purchased from Sigma-Aldrich Inc. (St. Louis, MO, USA), unless stated otherwise.

\section{Embryo collection and culture}

The ICR mice were fed ad libitum with a standard diet and were housed in a room with controlled temperature and lighting $\left(20-22^{\circ} \mathrm{C}, 12 \mathrm{~h} / 12 \mathrm{~h}\right.$ light/dark cycle), in accordance with the Institutional Animal Care and Use Committee (IACUC) guidelines of Nanjing Medical University. For the collection of zygotes, 8-week-old female ICR mice were injected with human chorionic gonadotropin (HCG) and mated with male ICR mice shortly after injection. After $21 \mathrm{~h}$, zygotes were collected from the oviducts of the mated female mice and transferred into 4-(2-hydroxyethyl)1-piperazineethanesulfonic acid (HEPES)-buffered Chatot, Ziomet and Bavister (CZB) medium. Cumulus cells were dispersed by treatment with $1 \mathrm{mg} / \mathrm{ml}$ hyaluronidase in HEPES-CZB. Cumulus-free zygotes were washed with HEPES-CZB medium and then cultured in CZB medium until they developed to later cleavage-stage embryos. All cultures were carried out within a humidified $5 \% \mathrm{CO}_{2}$ incubator at $37^{\circ} \mathrm{C}$.

\section{Immunofluorescence and confocal microscopy}

For immunofluorescence staining of ING2, the embryos were fixed in $4 \%$ paraformaldehyde in phosphate-buffered saline (PBS; pH 7.4) for at least 30 min at room temperature. After being permeabilized with $0.5 \%$ Triton X-100 at room temperature for 20 min, the embryos were incubated for $1 \mathrm{~h}$ in blocking solution composed of $1 \%$ bovine serum albumin in PBS, followed by incubation with goat anti-ING2 antibody (dilution, 1:100) overnight at $4^{\circ} \mathrm{C}$. After three washes in PBS containing 0.1\% Tween 20 and $0.01 \%$ Triton X-100 for 5 min each, the embryos were labelled with fluorescein isothiocyanate-conjugated rabbit anti-goat IgG (dilution, 1:100) for $1 \mathrm{~h}$ at room temperature. After three washes in PBS containing $0.1 \%$ Tween 20 and $0.01 \%$ Triton X-100, the embryos were co-stained with propidium iodide $(10 \mu \mathrm{g} / \mathrm{ml}$ in PBS), prior to being mounted on glass slides and examined under a confocal laser scanning microscope (Zeiss LSM 510 META, Jena Germany). 


\section{Immunoblotting analysis}

The extracted proteins of adult ICR mouse ovaries and morphologically normal 4-cell embryos (150 embryos/sample) were separated by sodium dodecyl sulfate polyacrylamide gel electrophoresis (SDS-PAGE) and then subsequently transferred to polyvinylidene fluoride membranes via electrophoresis. Following the transfer, the membranes were blocked in TBST (TBS buffer with $0.1 \%$ Tween 20) containing 5\% skimmed milk for $2 \mathrm{~h}$, followed by incubation overnight at $4^{\circ} \mathrm{C}$ with goat polyclonal anti-ING2 antibody (dilution, 1:500; sc-67646, Santa Cruz, Dallas, TX, USA). After being washed in TBST three times for $10 \mathrm{~min}$ each, the membranes were incubated for $1 \mathrm{~h}$ at $37^{\circ} \mathrm{C}$ with horseradish peroxidaseconjugated rabbit anti-goat IgG (dilution, 1:1000). Finally, the membranes were processed and analyzed using an enhanced chemiluminescence detection system (Amersham, Piscataway, NJ, USA).

\section{Reverse transcription polymerase chain reaction (RT-PCR)}

The primer sequences utilized for RT-PCR analysis of Ing2 expression were as follows: sense, 5'-GGGAGCTGGACAACACCTACCAA-3' and antisense, 5'-TTCGAGCATCTGGGTGACAATC-3'. Total RNA extraction and RT were performed using an RNeasy Micro Kit (Qiagen, Valencia, CA, USA), according to the manufacturer's instructions; with oligodT being utilized as a primer. Real-time PCR analysis was carried out using a Faststart Universal SYBR Green Master Mix (no. 04913914001, Roche Inc., Basel, Switzerland), with an ABI Prism 7500 System (Applied Biosystems, Foster City, CA, USA). We processed 1530 embryos at a time, and the H3f3a gene was utilized as an endogenous control (May et al., 2009). Relative quantitation of target gene expression was evaluated by the $2^{-\Delta \Delta C t}$ method (Livak \& Schmittgen, 2001) and data were collated from three replicate experiments utilizing different sets of embryos.

\section{ING2 MISSION ${ }^{\circledR}$ esiRNA and in vitro culture}

ING2 MISSION ${ }^{\circledR}$ esiRNA (EMU022101) and negative control EGFP MISSION ${ }^{\circledR}$ esiRNA (EHUEGFP) were purchased from Sigma-Aldrich Inc. (St. Louis, MO, USA), which possessed the intellectual property to design and prepare the MISSION esiRNA. The Ing2 cDNA target sequence is as follows: 5'-AAACGCCT ACAGCAGCATCTCCAGAGAGCGTTAATCAATAG CCAAGAATTGGGAGATGAAAAAATTCAGATTGT CACCCAGATGCTCGAATTGGTGGAGAACCGAGC GAGACAAATGGAGCTGCATTCACAGTGTTTCCA GGATCCTGCTGAAAGTGAGCGAGCCTCAGACA AGTCGAAGATGGATTCCAGTCAACCGGAAAGA
TCTTCTAGAAGACCTCGAAGACAGAGGACCAGT GAGAGCCGTGACTTATGTCACATGACAAACGGG ATTGACGACTGTGATGATCAACCACCGAAAGAA AAGAGATCCAAGTCCGCCAAGAAGAAGAAGC GCTCCAAGGCCAAGCAGGAGAGGGAGGCATCC CCTGTCGAGTTTGCCATCGATCCCAATGAGCCCA CCTACTGCTTGTGTAACCAAGTGTCCTACGGGG AGATGATAGGCTG-3'. MISSION esiRNA are a heterogeneous mixture of siRNAs that all target the same mRNA sequence. These multiple silencing triggers lead to lower off-target effects than single or pooled siRNAs, and exert highly specific and effective gene silencing. The concentration of ING2 MISSION esiRNA was $200 \mathrm{ng} / \mu \mathrm{l}$ in nuclease-free TE buffer (10 $\mathrm{mM}$ Tris-HCl, pH 8.0, 1 mM EDTA). Approximately $10 \mathrm{pl}$ of individual esiRNA was microinjected into the cytoplasm of the zygotes, which were cultured in CZB medium and subjected to further observation. The developmental progress of each group was observed and analyzed using a Nikon TE2000-S microscope.

\section{Statistical analysis}

Each experiment was repeated at least three times. Before statistical analysis, all percentage data were subjected to arc-sine transformation. Data were analyzed with one-way analysis of variance, paired Student's $t$-test and the chi-squared test, using SPSS software (SPSS Inc., Chicago, IL). Data are expressed as mean \pm standard deviation (SD), with a $P$-value $<$ 0.05 being considered statistically significant.

\section{Results}

\section{The expression of ING2 during mouse preimplantation development}

We analyzed the expression profile of Ing2 at each stage of mouse preimplantation embryonic development. The RT-PCR data confirmed expression of Ing2 in mouse preimplantation embryos (Fig. 1A), and further analysis revealed variation in expression levels of $I n g 2$ at different embryonic developmental stages (Fig. 1B). Most notably, Ing2 expression rapidly increased from the 2-cell to 4-cell cleavage-stage. This suggests that Ing 2 may be a crucial modulator of early embryonic development genes. Western blot analysis utilizing an anti-ING2 antibody revealed an exclusive band at the expected molecular mass of $33 \mathrm{kDa}$, for both the mouse ovary and 4-cell embryo (Fig. 2A).

\section{The localization of ING2 during mouse preimplantation development}

Laser scanning confocal microscope was used to analyze subcellular localization of ING2 protein at 

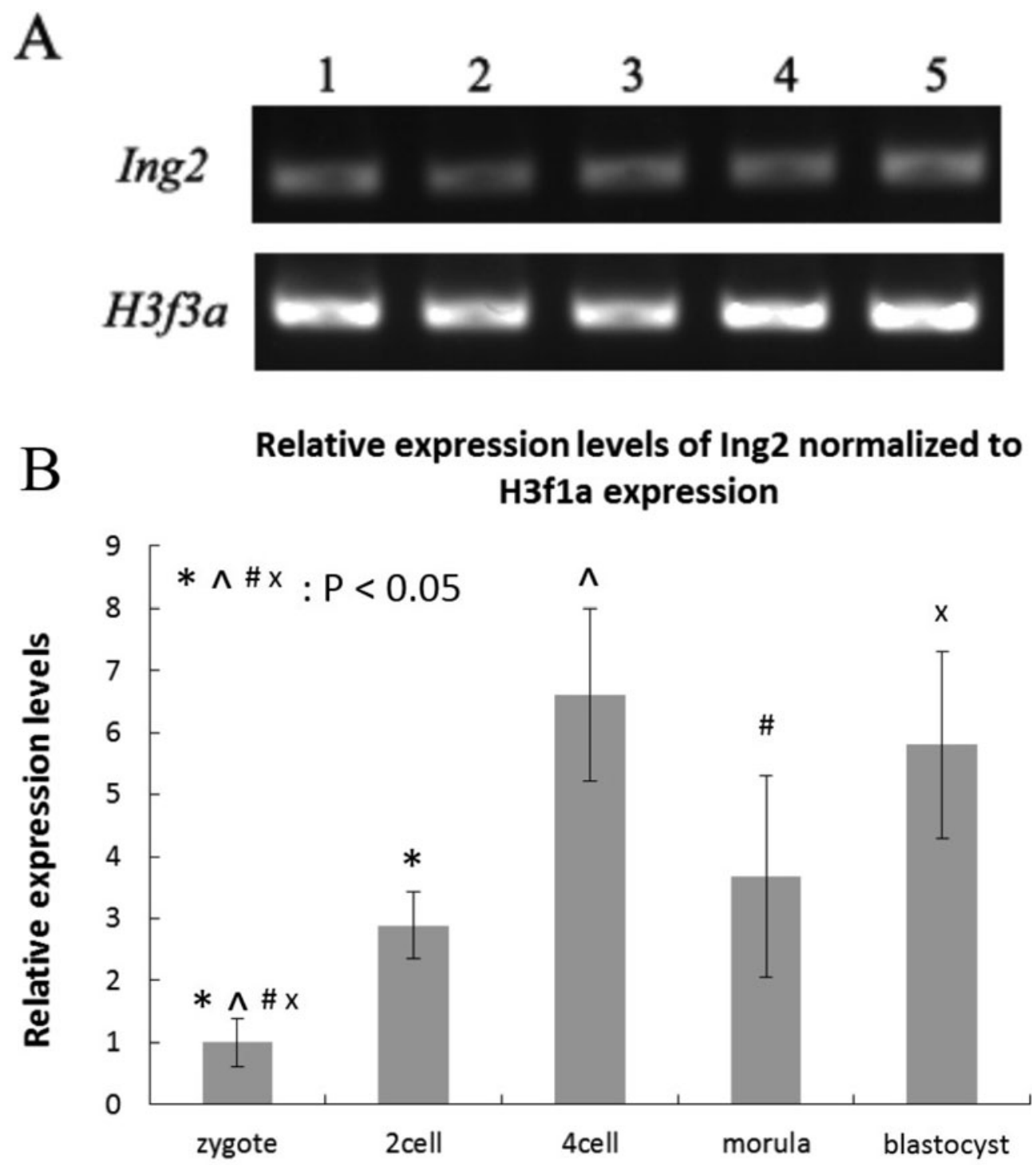

Figure $1(A)$ SDS-PAGE gel of RT-PCR amplification of Ing 2 mRNA transcripts extracted at different embryonic stages. H3f3a was utilized as the endogenous reference gene. Lanes 1, 2, 3, 4, 5 corresponds to zygote, 2-cell embryo, 4-cell embryo, morulastage embryo and blastocyst-stage embryo respectively. (B) Real-time PCR analysis of Ing 2 mRNA expression patterns at different embryonic stages during mouse preimplantation development. The expression level was calculated from the cycle threshold $\left(C_{t}\right)$ values by the $2^{-\Delta \Delta C t}$ method. The calibration sample was embryos at the zygote stage. Bar graphs indicate mean \pm standard deviation (SD) of three experimental replicates.

different stages of the mouse preimplantation embryo. There was increased expression of ING2 from the 2-cell embryo stage onwards with increasing accumulation within the nucleus beginning from the 4-cell embryo stage (Fig. 2B). Unstained 2-cell embryos were utilized as the negative controls (Fig. 2C). The immunostaining result is consistent with the mRNA expression patterns revealed by RT-PCR. ING2 C-terminal contains a nuclear localization sequence (NLS) domain, which possesses three nuclear targeting sequences (NTS) that target to the nucleolus. Hence, the nuclear localization of ING2 thus suggests that ING2 may have key functions in regulating gene transcription or chromosome modification.

\section{Knockdown of ING2 retards embryonic development in vitro}

To investigate the role of ING2 in mouse embryos, zygotes were microinjected with sigma MISSION esiRNA against ING2. Zygotes microinjected with sigma negative MISSION esiRNA served as controls. Real-time PCR showed that the expression level of ING2 was significantly reduced (Fig. $3 A$ ) upon 
Table 1 The developmental competence of embryos that were microinjected with ING2-specific esiRNA or negative esiRNA was evaluated by determining the ratios of microinjected embryos progressing to various developmental stages

\begin{tabular}{lcccc}
\hline & \multicolumn{4}{c}{ No. of embryos (\%) } \\
\cline { 2 - 5 } Treatment & Total & 4-cell & Morula & Blastocyst \\
\hline Ing2-esiRNA & 150 & $124(82.7)$ & $101(67.3)$ & $73(48.7)$ \\
Control & 132 & $125(94.7)^{* *}$ & $118(89.4)^{* *}$ & $100(75.8)^{* *}$ \\
\hline
\end{tabular}

** Values were considered significantly different from the control at $P<0.01$ (vs. control).

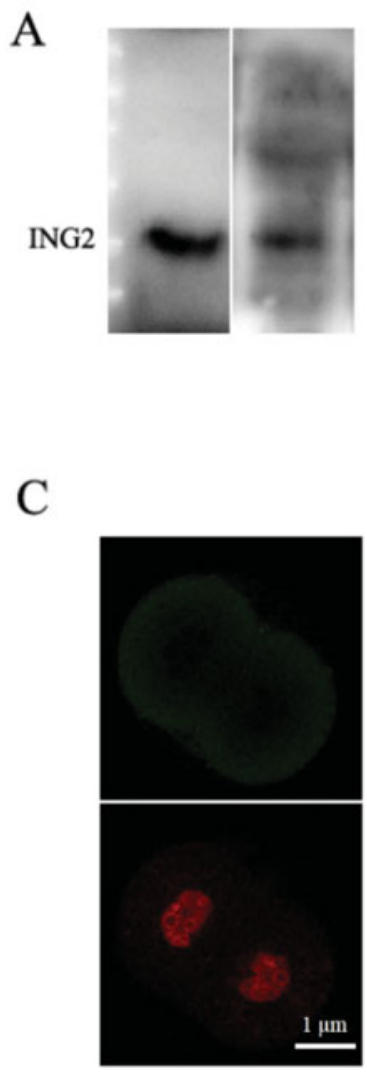

B

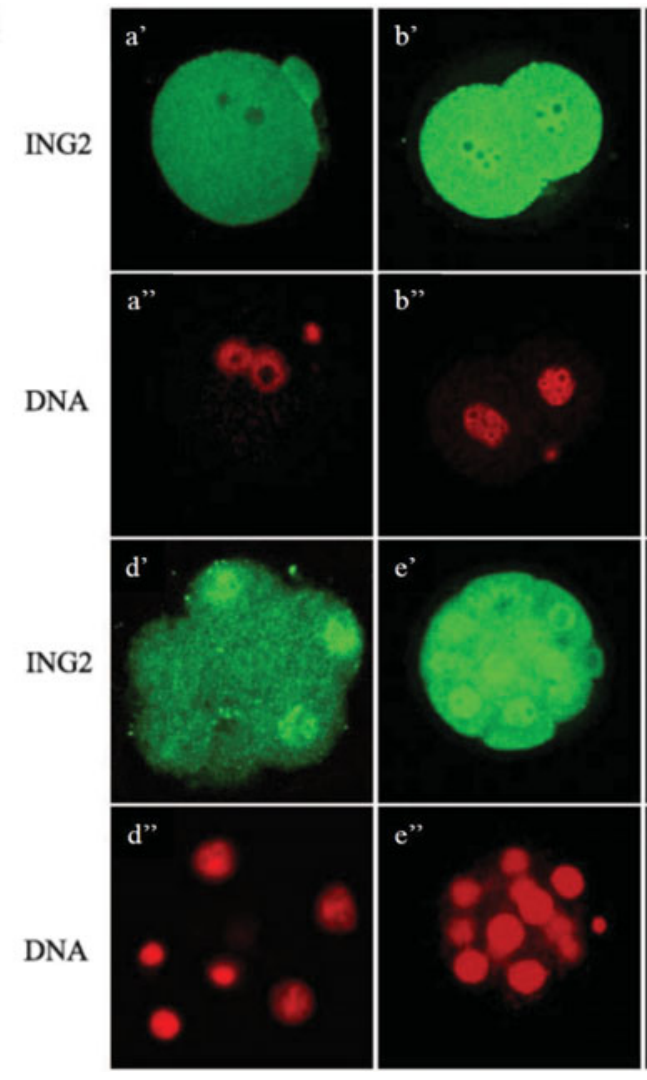

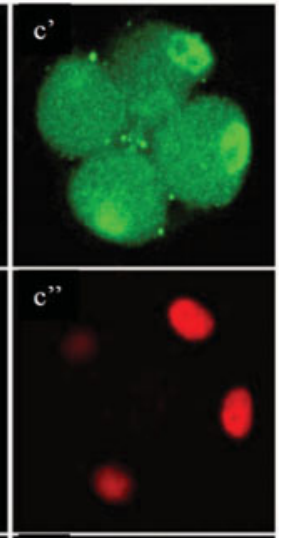
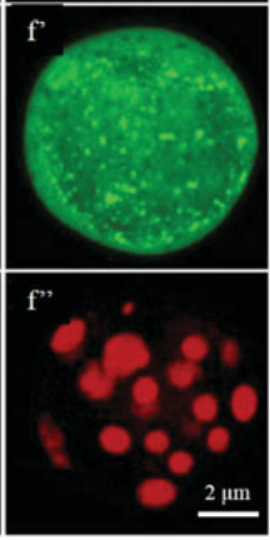

Figure $2(A)$ Western blot with the anti-ING2 antibody showed only one distinct band at the predicted molecular weight of ING2 protein $(33 \mathrm{kD})$, for both the mouse ovary and 4-cell embryo. (B) Immunofluorescence staining of ING2 and nuclear DNA in preimplantation embryos with specific anti-ING2 antibody (green, $a^{\prime}-f$ ) and propidium iodide $\left(\right.$ red, $\left.a^{\prime \prime}-f^{\prime}\right)$ respectively. zygote, approximately $21 \mathrm{~h}$ after human chorionic gonadotropin (HCG) injection $\left(a^{\prime}, a^{\prime \prime}\right)$; 2-cell embryo, approximately $45 \mathrm{~h}$ after HCG injection $\left(b^{\prime}, b^{\prime \prime}\right)$; 4- to 8-cell embryo, approximately $58 \mathrm{~h}$ after HCG injection $\left(c^{\prime}, c^{\prime \prime}, d^{\prime}, d^{\prime \prime}\right)$; morula, approximately $78 \mathrm{~h}$ after HCG injection (e, $\left.\mathrm{e}^{\prime \prime}\right)$ and blastocyst, approximately $96 \mathrm{~h}$ after HCG injection $\left(f, f^{\prime}\right)$. Scale bar: $2 \mu \mathrm{m}$. (C) Unstained 2-cell embryo was utilized as the negative control. Scale bar: $1 \mu \mathrm{m}$.

microinjection with the esiRNA, and this was further confirmed by reduced expression at the protein level detected by immunostaining (Fig. 3B). Inverted light microscopy was used to track the development of the treated embryos that were cultured up to the blastocyst stage (Fig. 3C). Notably, statistical analysis showed significant differences in the ratio of embryos that developed to the 4-cell, morula and blastocyst stage between the treated group $(n=150)$ and the negative control group $(n=132): 82.7 \%$ vs. $94.7 \%(P$
$<0.05), 67.3 \%$ vs. $89.4 \%(P<0.05), 48.7 \%$ vs. $75.8 \%$ $(P<0.01)$ (Table 1). This demonstrated that ING2 expression was essential to mouse preimplantation embryonic development.

\section{RNAi-mediated ablation of Ing2 results in increased p21 and decreased HDAC1 expression}

To gain a better understanding of the basis for the developmental delay of the Ing2-depleted embryos, we 
A
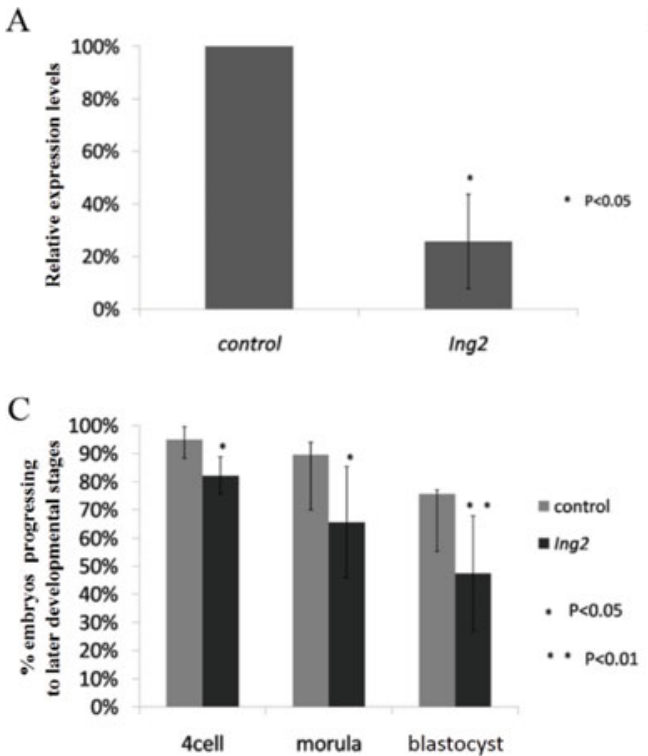

B
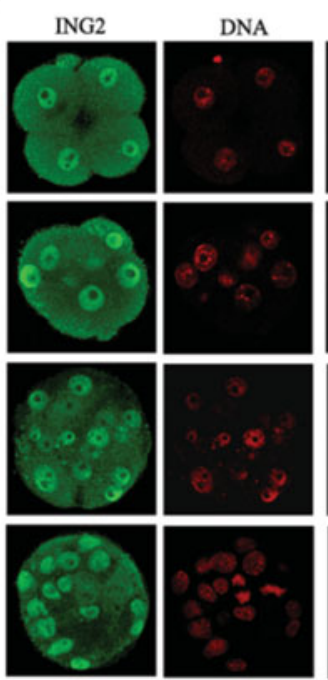

negtive esiRNA
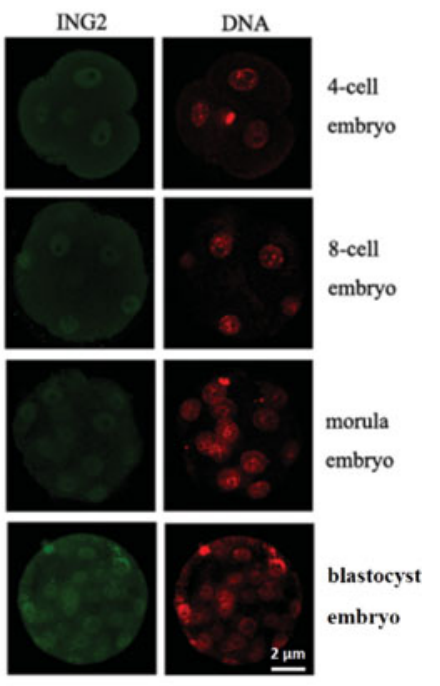

Ing2 RNAi

Figure 3 Zygotes were microinjected with ING2 specific sigma MISSION esiRNA. (A) Real-time PCR detected downregulation of Ing2 expression. The control and calibration sample was untreated zygotes. Error bars represent standard deviation $(\mathrm{SD})(* P<0.05)$. (B) Immunofluorescence staining confirmed decreased ING2 protein expression levels in the microinjected mouse embryos. (C) After zygotes were microinjected with ING2 specific esiRNA, the ratio of embryos that progressed to later embryonic developmental stages was evaluated. The depletion of ING2 led to developmental retardation. The results of five independent experiments were collated $(* P<0.05, * * P<0.01)$.

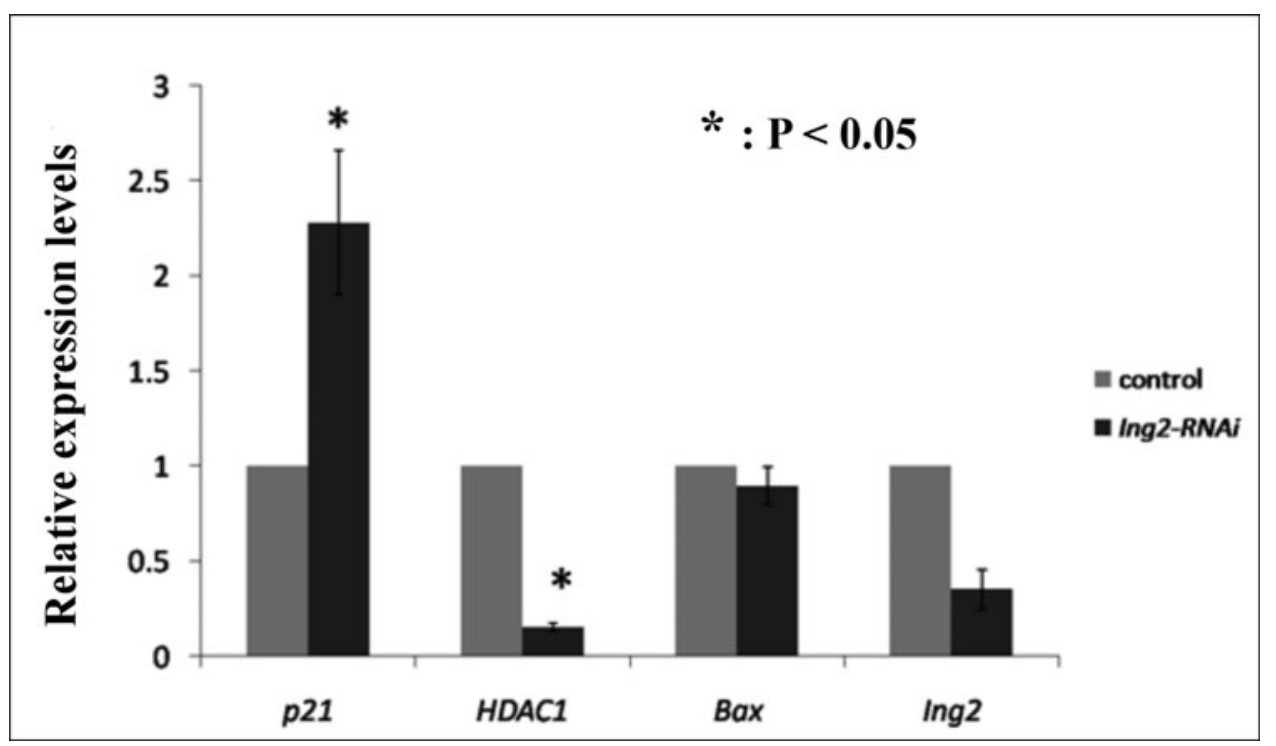

Figure 4 Real-time PCR analysis of expression of Ing2-associated genes in early blastocyst stage embryos after microinjection with ING2 specific esiRNA. The control and calibration sample was untreated embryos at the same developmental stage (early blastocyst). Data are presented as the mean of at least three biological replicates. Bar graphs indicate mean \pm standard deviation (SD); $* P<0.05$ vs. control.

assayed expression of $p 21, B a x$, and HDAC1 in these embryos by real-time PCR. The rationale for doing so is that ING2 activation leads to up-regulation of $p 21$ and Bax in a number of cancer cell lines (Nagashima et al., 2001). Additionally, HDAC inhibitors have been shown to induce expression of $p 21$ in many cell types and tumors, leading to cell cycle arrest (Zhu et al.,
2004). Induction of $p 21$ was also observed in Hdac1deficient embryonic stem cells (Lagger et al., 2002). In this study, we observed a 2.3-fold increase in $p 21$ mRNA expression level at the early blastocyst stage (96 $\mathrm{h}$ post-hCG injection), while there was a corresponding $85 \%$ decrease in HDAC1 mRNA level at the same time point in Ing2-depleted embryos (Fig. 4). It is 
hypothesized that the ING2-induced developmental delay could be due to decreased expression of $H D A C 1$ and concomitant increased expression of $p 21$, and may not be associated with apoptosis because there was no increase in the expression of the pro-apoptotic Bax transcripts (Fig. 4).

\section{Discussion}

In this study, we investigated the expression and localization of ING2 during mouse preimplantation development. We also demonstrated its physiological function in embryonic development and made preliminary investigations on its mechanism of action.

RT-PCR analysis revealed that there was a marked increase in Ing2 expression from the 2-cell to 4cell embryo stage. This is consistent with the immunostaining results, which showed an increase in expression of ING2 at the protein level from the 2cell embryo stage, together with increasing localization within the nucleus of 4-cell blastomeres. In mouse, the first upsurge of gene expression occurs at the 2cell embryo stage, and is known as zygotic genome activation (ZGA) (Flach et al., 1982; Latham et al., 1992; Nothias et al., 1995). From then onwards, the embryo will exploit its own transcriptional and translational machinery to carry out its developmental program. This enables reprogramming of the newly formed embryonic genome to a totipotent state, which is subsequently lost at the blastocyst stage (Duranthon et al., 2008; Calle et al., 2012). Previous research suggested that genes activated from the transition of fertilized eggs to 4-cell embryos contribute mainly to the regulation of basic cellular machinery, while genes activated from transition of 8-cell embryos to the morula and blastocyst stages are mainly associated with dramatic biological and morphological events (Hamatani et al., 2004). The spatio-temporal expression pattern of ING2, may give some cues on how it may contribute to mouse preimplantation development. Further investigation showed that down-regulation of ING2 by RNAi led to a decreased blastocyst formation rate compared with the negative control group, which means that ING2 does play a crucial role in preimplantation development.

As a growth inhibition factor, ING2 has a PHD domain that enables its interaction with $\mathrm{H} 3 \mathrm{~K} 4 \mathrm{Me} 3$ to regulate gene repression (Gozani et al., 2003; Shi et al., 2006). ING2 is a stable component of the Sin3-HDAC complex. It has been shown that ING2 negatively regulates cell proliferation, chromatin remodeling, apoptosis and DNA repair through modulation of the p53 acetylation pathway, which incidentally enhance its own transcription (Wang et al., 2006; Unoki et al.,
2008). Additionally, ING2 has also been implicated in muscle differentiation through regulation of myogenin transcription (Eapen et al., 2012). The tri-methylated histone mark is found mainly in transcriptionally active regions (Bannister \& Kouzarides, 2004). ING2, being a stable component of the Sin3-HDAC complex, recruits and stabilizes the Sin3-HDAC complex on gene promoters, leading to histone deacetylation and gene repression (Doyon et al., 2006; Pena et al., 2006; Shi et al., 2006). Histone deacetylase (HDAC) inhibitors cause the dissociation of the PHD domain containing ING2 subunit from the Sin3 deacetylase complex. The loss of ING2 subsequently disrupts the in vivo binding of the Sin 3 complex to the p21 promoter (Smith et al., 2010). In this study, we found that $p 21$ expression was significantly increased in ING2 knockdown embryos. Additionally, there was dramatically decreased expression of HDAC1 in these ING2 knockdown embryos. These results thus suggest that $p 21$ as well as HDAC1 may contribute to the regulation of preimplantation embryonic development. Previous studies show that down-regulation of HDAC1 leads to hyperacetylation of histone $\mathrm{H} 4$, which in turn results in developmental delay. The underlying molecular mechanism involves increased expression of $p 21$, without any increase in expression of either the pro-apoptotic Bax or $B c l 2$ transcripts within the HDAC1-depleted embryos (Ma \& Schultz, 2008). Additionally, induction of p21 was also observed in Hdac1-deficient embryonic stem cells (Lagger et al., 2002). In summary, this study has demonstrated that ING2 plays important roles in mouse preimplantation development which may be associated with complex epigenetic mechanisms.

Nevertheless, it must be noted that although our results do show that ING2 plays a crucial role in preimplantation development, it is unlikely to be the unique modulator of this process. As seen in Fig. 3C and Table 1, only a certain percentage of embryos are affected by microinjection of ING2-specific esiRNA. This could suggest the existence of multiple redundant signalling pathways in preimplantation development. Indeed, it has been hypothesized that this redundancy may provide a fail-safe protection to the preimplantation development programme (Kaye, 1997).

As a basic subunit of the Sin3-HDAC complex, ING2 displays a high degree of interspecies homology between human and mouse (Guerillon et al., 2013). Hence, we hypothesize that ING2 may also play a similar key role in human preimplantation embryonic development. In clinical assisted reproduction, obtaining high-quality embryos is critical for improving the pregnancy rate. Additionally, extended culture and transfer of embryos at the blastocyst stage for assisted reproduction offers some theoretical advantages over the transfer of cleavage-stage embryos. For example, higher implantation rates, single embryo transfer and 
better temporal synchronization between embryo and endometrium can be achieved by transfer of embryos at the blastocyst stage (Jones et al., 1998; Tsirigotis, 1998; Hambiliki et al., 2013). However, the extended duration of in vitro culture involves high risk of reduction in the number of embryos available for transfer, because the in vitro culture environment may exert detrimental effects on embryonic development (Jones et al., 1998; Kawamura et al., 2012; Calzi et al., 2012). Hence rigorous characterization of the regulatory mechanisms in embryonic development is of great significance to clinical assisted reproduction. This study sheds some light on the crucial role of ING2 in mouse preimplantation development which gives an inkling on the function of ING2 in human in vitro cultured embryos.

\section{Competing interests}

The authors hereby declare that there are no conflicting interests.

\section{Acknowledgements}

This work was financially supported by the National Natural Science Foundation of China (81100420, 81270701).

\section{References}

Ahringer, J. (2000). NuRD and SIN3 histone deacetylase complexes in development. Trends Genet. 16, 351-6.

Bannister, A.J. \& Kouzarides, T. (2004). Histone methylation: recognizing the methyl mark. Methods Enzymol. 376, 26988.

Braude, P., Bolton, V. \& Moore, S. (1988). Human gene expression first occurs between the four- and eightcell stages of preimplantation development. Nature 332, $459-61$.

Calle, A., Fernandez-Gonzalez, R., Ramos-Ibeas, P., LagunaBarraza, R., Perez-Cerezales, S., et al. (2012). Long-term and transgenerational effects of in vitro culture on mouse embryos. Theriogenology 77, 785-93.

Calzi, F., Papaleo, E., Rabellotti, E., Ottolina, J., Vailati, S., et al. (2012). Exposure of embryos to oxygen at low concentration in a cleavage stage transfer program: reproductive outcomes in a time-series analysis. Clin. Lab. 58, 997-1003.

Dal Canto, M., Coticchio, G., Mignini Renzini, M., De Ponti, E., Novara, P.V., et al. (2012). Cleavage kinetics analysis of human embryos predicts development to blastocyst and implantation. Reprod. Biomed. Online 25, 474-80.

Doyon, Y., Cayrou, C., Ullah, M., Landry, A.J., Cote, V., et al. (2006). ING tumor suppressor proteins are critical regulators of chromatin acetylation required for genome expression and perpetuation. Mol. Cell 21, 51-64.
Duranthon, V., Watson, A.J. \& Lonergan, P. (2008). Preimplantation embryo programming: transcription, epigenetics, and culture environment. Reproduction 135, 14150.

Eapen, S.A., Netherton, S.J., Sarker, K.P., Deng, L., Chan, A., et al. (2012). Identification of a novel function for the chromatin remodeling protein ING2 in muscle differentiation. PLoS One 7, e40684.

Ecker, D.J., Stein, P., Xu, Z., Williams, C.J., Kopf, G.S., et al. (2004). Long-term effects of culture of preimplantation mouse embryos on behavior. Proc. Natl. Acad. Sci. USA 101, 1595-600.

Flach, G., Johnson, M.H., Braude, P.R., Taylor, R.A. \& Bolton, V.N. (1982). The transition from maternal to embryonic control in the 2-cell mouse embryo. EMBO J. 1, 681-6.

Gardner, D.K., Vella, P., Lane, M., Wagley, L., Schlenker, T., et al. (1998). Culture and transfer of human blastocysts increases implantation rates and reduces the need for multiple embryo transfers. Fertil. Steril. 69, 84-8.

Goeman, F., Otto, K., Kyrylenko, S., Schmidt, O. \& Baniahmad, A. (2008). ING2 recruits histone methyltransferase activity with methylation site specificity distinct from histone $\mathrm{H} 3$ lysines 4 and 9. Biochim. Biophys. Acta 1783, 1673-80.

Gozani, O., Karuman, P., Jones, D.R., Ivanov, D., Cha, J., Lugovskoy, A.A., Baird, C.L., Zhu, H., Field, S.J., Lessnick, S.L., Villasenor, J., Mehrotra, B., Chen, J., Rao, V.R., Brugge, J.S., Ferguson, C.G., Payrastre, B., Myszka, D.G., Cantley, L.C., Wagner, G., Divecha, N., Prestwich, G.D., \& Yuan, J. (2003). The PHD finger of the chromatin-associated protein ING2 functions as a nuclear phosphoinositide receptor. Cell 114, 99-111.

Guerillon, C., Larrieu, D. \& Pedeux, R. (2013). ING1 and ING2: multifaceted tumor suppressor genes. Cell. Mol. Life Sci. 70, 3753-72.

Hamatani, T., Carter, M.G., Sharov, A.A. \& Ko, M.S. (2004). Dynamics of global gene expression changes during mouse preimplantation development. Dev. Cell 6, 11731.

Hambiliki, F., Hanrieder, J., Bergquist, J., Hreinsson, J., Stavreus-Evers, A., et al. (2013). Glycoprotein 130 promotes human blastocyst development in vitro. Fertil. Steril. 99, 1592-9.

Jones, G.M., Trounson, A.O., Gardner, D.K., Kausche, A., Lolatgis, N., et al. (1998). Evolution of a culture protocol for successful blastocyst development and pregnancy. Hum. Reprod. 13, 169-77.

Kawamura, K., Chen, Y., Shu, Y., Cheng, Y., Qiao, J., et al. (2012). Promotion of human early embryonic development and blastocyst outgrowth in vitro using autocrine/paracrine growth factors. PLoS One 7, e49328.

Kaye, P.L. (1997) Preimplantation growth factor physiology. Rev. Reprod. 2, 121-7.

Lagger, G., O'Carroll, D., Rembold, M., Khier, H., Tischler, J., et al. (2002). Essential function of histone deacetylase 1 in proliferation control and CDK inhibitor repression. EMBO J. 21, 2672-81.

Larrieu, D., Ythier, D., Binet, R., Brambilla, C., Brambilla, E., et al. (2009). ING2 controls the progression of DNA replication forks to maintain genome stability. EMBO Rep. 10, 1168-74. 
Latham, K.E., Solter, D. \& Schultz, R.M. (1992). Acquisition of a transcriptionally permissive state during the 1-cell stage of mouse embryogenesis. Dev. Biol. 149, 457-62.

Livak, K.J. \& Schmittgen, T.D. (2001) Analysis of relative gene expression data using real-time quantitative PCR and the $2^{-\Delta \Delta \mathrm{CT}}$ method. Methods 25, 402-8.

Ma, P. \& Schultz, R.M. (2008). Histone deacetylase 1 (HDAC1) regulates histone acetylation, development, and gene expression in preimplantation mouse embryos. Dev. Biol. 319, 110-20.

May, A., Kirchner, R., Muller, H., Hartmann, P., El Hajj, N., et al. (2009). Multiplex RT-PCR expression analysis of developmentally important genes in individual mouse preimplantation embryos and blastomeres. Biol. Reprod. 80, 194-202.

Monk, M., Boubelik, M. \& Lehnert, S. (1987). Temporal and regional changes in DNA methylation in the embryonic, extraembryonic and germ cell lineages during mouse embryo development. Development 99, 371-82.

Nagashima, M., Shiseki, M., Miura, K., Hagiwara, K., Linke, S.P., et al. (2001). DNA damage-inducible gene p33ING2 negatively regulates cell proliferation through acetylation of p53. Proc. Natl. Acad. Sci. USA 98, 9671-6.

Niemann, H. \& Wrenzycki, C. (2000). Alterations of expression of developmentally important genes in preimplantation bovine embryos by in vitro culture conditions: implications for subsequent development. Theriogenology $53,21-34$.

Nothias, J.Y., Majumder, S., Kaneko, K.J. \& De Pamphilis, M.L. (1995). Regulation of gene expression at the beginning of mammalian development. J. Biol. Chem. 270, 22077-80.

Pedeux, R., Sengupta, S., Shen, J.C., Demidov, O.N., Saito, S., et al. (2005). ING2 regulates the onset of replicative senescence by induction of p300-dependent p53 acetylation. Mol. Cell. Biol. 25, 6639-48.

Santos, F., Peters, A.H., Otte, A.P., Reik, W. \& Dean, W. (2005). Dynamic chromatin modifications characterise the first cell cycle in mouse embryos. Dev. Biol. 280, 225-36.

Schultz, R.M. (2005). From egg to embryo: a peripatetic journey. Reproduction 130, 825-8.
Schultz, R.M. \& Williams, C.J. (2002). The science of ART. Science 296, 2188-90.

Shi, X., Hong, T., Walter, K.L., Ewalt, M., Michishita, E., et al. (2006). ING2 PHD domain links histone H3 lysine 4 methylation to active gene repression. Nature 442, 96-9.

Smith, K.T., Martin-Brown, S.A., Florens, L., Washburn, M.P. \& Workman, J.L. (2010). Deacetylase inhibitors dissociate the histone-targeting ING2 subunit from the Sin 3 complex. Chem. Biol. 17, 65-74.

Torres-Padilla, M.E., Bannister, A.J., Hurd, P.J., Kouzarides, T. \& Zernicka-Goetz, M. (2006). Dynamic distribution of the replacement histone variant $\mathrm{H} 3.3$ in the mouse oocyte and preimplantation embryos. Int. J. Dev. Biol. 50, 455-61.

Tsirigotis, M. (1998). Blastocyst stage transfer: pitfalls and benefits. Too soon to abandon current practice? Hum. Reprod. 13, 3285-9.

Unoki, M., Kumamoto, K., Robles, A.I., Shen, J.C., Zheng, Z.M., et al. (2008). A novel ING2 isoform, ING2b, synergizes with ING2a to prevent cell cycle arrest and apoptosis. FEBS Lett. 582, 3868-74.

Wang, Y., Wang, J. \& Li, G. (2006). Leucine zipper-like domain is required for tumor suppressor ING2-mediated nucleotide excision repair and apoptosis. FEBS Lett. 580, 3787-93.

Watson, A.J. \& Barcroft, L.C. (2001). Regulation of blastocyst formation. Front. Biosci. 6, D708-30.

Yamanaka, Y., Ralston, A., Stephenson, R.O. \& Rossant, J. (2006). Cell and molecular regulation of the mouse blastocyst. Dev. Dynam. 235, 2301-14.

Yeo, S., Lee, K.K., Han, Y.M. \& Kang, Y.K. (2005). Methylation changes of lysine 9 of histone $\mathrm{H} 3$ during preimplantation mouse development. Mol. Cell. 20, 423-8.

Zhang, H.K., Pan, K., Wang, H., Weng, D.S., Song, H.F., et al. (2008). Decreased expression of ING2 gene and its clinicopathological significance in hepatocellular carcinoma. Cancer Lett. 261, 183-92.

Zhu, P., Huber, E., Kiefer, F. \& Gottlicher, M. (2004). Specific and redundant functions of histone deacetylases in regulation of cell cycle and apoptosis. Cell Cycle 3, 1240-2. 